\title{
"Community" and "Compassion": Research on the Charity Donation Behavior of Fans of the Cultivation Department
}

\author{
Lei Qian ${ }^{1, a^{*}}$ \\ ${ }^{1}$ School of Communication, Fujian Normal University, Fuzhou, China \\ $a^{*}$ Lqian_2001@163.com

\begin{abstract}
Fans of the Cultivation Department donate to public disasters in the name of stars through the Internet platform. The large amount of fundraising and the strong ability to act have aroused widespread concern in the society. This paper studies the mechanism behind such public welfare behaviors of fans, using the research methods of online ethnography and in-depth interviews, and analyzing the collected materials through thematic analysis methods. The research found that: 1) Due to the role of "bridges" in the development of celebrities, fans A "community" network was formed around the stars of the cultivating system. Because of the existence of the core of being a celebrity, fans can express their individual demands or behaviors in a "community" way and turn them into collective actions. 2) With the support of the "community" network, empathy makes collective actions more emotional than individual actions. The two mechanisms of "community" and "empathy" finally realized the transfer of the identity of nurturing fans from the private sphere to the public sphere, thus contributing to the realization of charity donations.
\end{abstract}

Keywords: Community, empathy, identity, public domain, transformation mechanism

\section{“共同体”与 “共情”: 养成系粉丝公益捐款行为研究}

\section{雷茜 ${ }^{1,} \mathrm{a}^{*}$}

${ }^{1}$ 福建师范大学传播学院, 福州, 中国

$a^{a^{*}}$ Lqian_2001@163.com

摘要

养成系粉丝通过互联网平台以明星的名义为公共灾害捐款, 其筹款金额之大和行动力之强引起了社会的广泛关 注。本文研究粉丝此类公益行为背后的机制, 采用网络民族志和深度访谈的研究方法, 通过主题分析的方法分 析所收集的材料，研究发现：1）由于养成系明星的 “桥梁” 的作用，粉丝围绕在养成系明星周围形成了 “共同 体” 网络。因为养成系明星这个核心的存在，粉丝可以把个人诉求或行为以 “共同体” 的方式表达出来而变成 集体行动。2)在 “共同体” 网络的支持下，共情使得集体行动比个人行为具有更强烈的情感。“共同体” 和 “共 情” 两种机制最终实现了养成系粉丝的身份认同由私人领域转入公共领域，从而促成了公益捐款的实现。 关键词: 共同体; 共情; 身份认同; 公共领域; 转型机制

\section{1. 研究背景}

2020 年新冠肺炎疫情爆发期间，养成系粉丝通 过各种各样的互联网平台进行捐款, 其筹款金额和行 动力引起了社会的关注。相较于传统类明星来说, 以 “网络养成” 为标签的 “养成系” 明星的粉丝参与慈
善捐款的行为在微博上引起了不小的影响。以 TFBOYS 为例, 据不完全统计, 易烊千罕 40 个粉丝站的其中 12 个粉丝站, 在此次疫情期间捐款捐物超过了 141 万元。王源粉丝联合（各粉丝站+粉丝自发）总计捐 赠金额 407048. 85 元。王俊凯粉丝也陆续捐款累计 53 万多元。7 月初南方经历连续暴雨, 灾情严重, @ 
易烊千胥 V 公益发布 “抗洪救灾公益”，据统计，7 月 10 日 9 时至 13 时共四小时整, 参与捐款人数达 2986 人，累计捐款 256876 元。展现出了易烊千胥粉 丝极其强大的行动力。虽然 2021 年 5 月初, 《青春有 你 3》节目的 “倒牛奶事件” 引发大众关注。粉丝通 过谁买的奶多、谁扫的卡多为喜欢的偶像证明人气， 粉丝只要瓶盖不要奶, 这样的行为被认为是“狂热的” “盲目的”, 他们背后的情感是 “患蚌的” “危险的”。 但是正如上文所说，他们的行为也同样会导向对于社 会有益的方向。那么, 养成系粉丝与传统粉丝不同, 为什么他们会因为喜欢某一个养成系明星而促使他 们以这个明星的名义去捐款? 这个现象背后呈现出 一种怎样的机制? 本研究将要探讨粉丝的私人行为 转化为对公共有利的行为其中的机制, 这个过程是如 何发生的? 以此我们希望通过理解这样一种过程和 行为发生的机制, 能够帮助、促进、引导粉丝更多地、 更好地向对公共有益的方向发展。

\section{2. 作为公众的粉丝}

哈贝马斯指出: “所谓 “公共领域”, 我们首先意 指我们的社会生活的一个领域, 在这个领域中, 像公 共意见这样的事物能够形成。[1]”这样的公共领域 是对所有公民开放的, 作为个体的人们集合在一起而 成为公众, 他们就共同关心的问题进行对话和讨论 [2]。中国式公共领域的根本目的在于实现中国社会 的良性运行以及现代化的目标, 所传递的应该是社会 中负责任的公民的理性声音, 应该是在理性基础上的 共识达成 [2]。作为一种限制与区分, 谋生需要和生 活事宜被指定在私人领域 [3]。在流行观点和大众媒 体中, 粉丝常常呈现出一种负面形象, 他们常被刻画 为原子化的、受到操控的、沉迷的且不理性的个体, 在很多方面都与群众（就狂热和情绪化而言）和大众 （就关注点重合以及缺乏沟通而言）相似 [4]。

因此, 粉丝的不正当、不理智行为是属于私人领 域的行为。这些行为使得在公众的印象中, 粉丝的形 象与 “脑残粉”、“盲目”、“疯子” 等关键词挂钩, 而 粉丝群体因为对所喜欢的明星的情感转化成做出对 公共社会有益处的事情的行为属于公共领域的行为。 相对于传统的明星来说, 养成系明星的粉丝相对于传 统的明星的粉丝来说具有更强烈的情感, 他们的行为 也可能因此更加 “盲目”、“疯狂”。“养成” 系明星是 指由娱乐公司打造, 以 “培养”、“陪伴成长” 为诉求 点, 贩卖自己真实的人生成长过程, 使粉丝诉诸于自 身 “参与感” 和 “认同感”，从而付出时间、资金、 感情来制造的一类明星 [5]。对于养成类明星的粉丝 而言, 在陪伴偶像成长, 看着他们不断努力产生蜕变 过程中，付出时间、感情和金钱, 无形中将潜意识中 的 “自我” “投射” 到偶像身上, 不断完善自我认知, 对自己的价值观产生确认感和认同感 [6]。粉丝在对 养成系明星相关的文本创作的传播和共享中, 展示了 自己作为粉丝在 “养成” 过程中付出的心血和经历, 证明自己在这个群体的影响, 从而产生的归属感、满
足感和认同感在信息与知识的共享中得到强化, 并且 在这个过程中，越来越多的新粉丝会因为这些粉丝文 本的生产而加入集体, 获得认同感的粉丝会更有动力 进行创作, 确认自己的价值, 在群体中找到自己的定 位和归属, 从而形成群体内部认同 [6]。他们通过文 本创作的形式让新粉加入, 此行为就是在 “公共领域” 下进行的, 因此他们不再是 “私人”, 是作为 “公众” 的粉丝。

张玮玉在《粉丝公众》一书中提到: “只有当共 同关注特定议题的个体努力通过参与彼此间和与其 他社会成员的讨论, 从而在更大的社会范围内代表自 己时候, 公众才会形成。因此, 当粉丝在试图表现他 们的兴趣并且将他们的诉求展现在更广大的社会面 前时，他们是可以成为公众的。[4]” 过去的学者对 于粉丝在饭圈内部的私人领域的身份认同已经做了 充分的研究, 但是不难发现, 上述的研究中的身份认 同更多是在饭圈范围内, 属于私人领域的范围, 少数 将身份认同与公共领域联系并导向粉丝积极的行为 中, 且并未深入研究粉丝作为公众在公共领域的视角 下的身份认同，因此本文将研究粉丝作为公众在公共 领域下所产生的积极的行为。

\section{3. 粉丝的社会共情}

而粉丝常被认为是不理性的，而是情绪性的，因 为他们通过不同的媒介进行沟通, 对他们所喜欢的偶 像投入了持久的感情。虽然从理论上, 粉丝因为缺乏 “理性” 这一核心特征而无法作为公众, 但在我们生 活中, 非理性的表达已经被广泛应用于公共讨论 [4]。 然而, 情感与公共领域的运作息息相关, 尤其在互联 网群体的传播中, 传播是一种情感名义下的传播 [7]。 现实中有些关系到集体和社会价值的问题需要情感 的介入才能够得到尽善尽美的解决 [7]。所以我们可 以松开其常规意义上的约束条件，包容地看待对于 “公众” 的定义 [4]。粉丝作为个体, 他们共同关心 所喜欢的明星，彼此之间进行对话和讨论时产生某种 集体性的情感经验, 有导向某种公共性行为的可能性。

从生活经验方面来看, 在现实社会中, 情感也包 含理性。而在过去的研究中, 公共领域与 “理性” 因 素相结合, 私人领域与 “感性” 因素相结合都已经讨 论得很充分了。而粉丝作为公众, 他们的情感和理性 是分不开的, 情感包含了理性的维度。所以为了填补 这一研究空白, 本研究将结合 “理性” 和 “感性” 两 种情感因素, 探究情感机制是如何让粉丝的行为从饭 圈的私人领域扩展到其他的公共领域的, 如何将粉丝 仅仅是对明星的喜爱拓展到对公益的追求的, 将个人 情感上升至对社会的贡献。

\section{4. 由私到公的领域转型机制}

孙雨田在对 “养成” 系明星的粉丝文化进行研究 时, 全面详细的研究了以 TFBOYS 为例的养成系明星 的身份认同。她认为粉丝个体在自我肯定中实现的自 
我认同，在与他者的互动中实现群内认同，在群体互 动中实现群体认同, 这种群体认同包括在粉丝群体内 部构建 “小社会”、在与反迷群的博弯中形成认同和 迷群与社会大众形成互动 [5]。蔡叶枫同样对以 TFBOYS 为例的养成系明星的身份认同进行了详细的 研究。她认为粉丝在个体的实践中实现自我认同，在 与他者互动中形成群体认同, 在群体互动中实现群体 认同 [6]。张玮玉在研究粉丝时, 打破原有的界定, 将粉丝认为是公众, 认为他们围绕特定的议题与其他 成员进行讨论 [4], 其实已经将粉丝的行为认定为在 公共领域下进行的, 但是为什么粉丝会从私人领域转 向公共领域, 这其中的转型机制有待深入研究。

过去粉丝很少作为公众被放在公共领域进行讨 论, 但易烊千胥的粉丝在此次疫情期间以其名义进行 捐款, 表现出了他们对于这一方面的兴趣, 并且将这 种诉求展现在了更广大的社会面前, 所以应当将他们 的行为从私人领域提升到公共领域进行讨论。上述两 位学者在对粉丝的身份认同的研究都较为详细且全 面, 研究中将身份认同与社会、大众构建联系, 可以 认为其已被在公共领域下进行讨论, 但并未深入研究 身份认同让粉丝的行为从私人领域转向公共领域的 逻辑过程, 其中包含了怎样的一种转型机制。

所以, 本研究将重点探究养成系明星的粉丝作为 公众他们的身份认同将导向什么? 探究情感机制是 如何让粉丝的行为从饭圈的私人领域扩展到其他的 公共领域的?

\section{5. 研究方法}

首先选取养成系明星的代表 TFBOYS, TFBOYS 诞 生于互联网, 也通过互联网发展和走红, 三人具有强 大的粉丝团, 在微博上的粉丝数量均为 8000 多万。 其次, 选取 TFBOYS 团体中年龄最小的一名成员易烊 千胥, 从一开始粉丝最少、知名度最低到如今在微博 拥有 8500 多万的粉丝, 也为宝马、蒂芙尼、宝格丽 等高端奢侈品牌代言。所以选取 TFBOYS 中的易烊千 尔及其粉丝 “千纸鹤” 作为研究对象。

微博, 强化了粉丝与名人间的即时和常态的互动, 对许多普通中国人而言, 它创造出一种新型社会关系, 这种新型关系承载了信息、情绪和情感的流动, “粉 丝一一名人” 网络不仅传递着名人的表现和粉丝的 反应, 并且名人在各类议题中的表现令其成为连接粉 丝和这些议题的桥梁 [4]。名人在此次疫情这类议题 中积极宣传, 并且积极参与疫情的捐款, 成为连接粉 丝和这些议题的桥梁, 并且能够有效地吸引、鼓励粉 丝也参与到这件事当中去, 而微博在这其中扮演了很 重要的作用。并且微博是 TFBOYS 粉丝数量最多、参 与度最高的社交平台, 所以选取微博作为研究平台。

首先, 本文主要采用以下两种研究方法:

1. 网络民族志 (virtual ethnography): 在新浪 微博直接搜索 “易烊千胥公益”, 可以看到众多用户
在此平台发布关于易烊千玺参与公益的记录。其中最 显眼的便是 “易烊千胥 V 公益” 这个微博用户，点击 进入该用户主页可以发现, 在此用户中, “千纸鹤” 们可以选择他们力所能及能够参与的公益, 也有为受 到自然灾害的地区募捐的通道, 后续该用户会对每一 笔资金的流动进行公示，展现出了 “公开化”、“透明 化” 的特征。通过对微博平台中的研究对象的语言、 行为进行参与式观察, 民族志记录, 并结合文献进行 探析和诠释。

2. 深度访谈: 通过滚雪球的方式在微博中选取部 分粉丝进行深度访谈, 深度了解他们个人与易烊千坌 之间的态度和情感关系, 从而深入研究养成系明星与 粉丝之间在新型社会关系中所形成的身份认同是如 何导致粉丝的行为从私人领域转向公共领域的。

其次，运用主题分析的方法分析了所收集的资料。 本文分析的话语主要有两种类型: 一是网络民族志期 间观察到的易烊千筀粉丝在参与捐款的整个过程中 在微博上发表的文本和评论, 以及民族志记录。二是 深度访谈后整理出的逐字稿。通过使用主题分析的方 式，分析粉丝之间是如何产生身份认同，这种身份认 同是如何使得他们从私人领域转向公共领域的。

\section{6. 研究发现}

\section{1. 公共领域下身份认同与 “共同体” 的形 成}

养成系明星与粉丝之间的关系已经与传统明星 与粉丝之间的关系不同, 他们在社交平台公开自己的 生活, 把自己的真实人生成长过程展现给大众, 他们 与粉丝之间依托社交媒体实现双向互动, 粉丝可以真 切地见证偶像的成长, 更直接地参与到偶像的生活当 中, 这种 “参与感” 使得粉丝群体与养成系明星之间 形成了身份认同，这种身份认同加强了粉丝与明星之 间的黏性，使得养成系明星相较于传统类明星与粉丝 之间具有一种更强的信任。粉丝作为一个群体, 他们 是公众, 他们的行为很多情况下也是在公共领域所进 行的。当粉丝群体为了一个公共事件行动时, 他们便 成为公众, 特别是粉丝在为社会大众普遍关注的事件 做贡献, 且为社会带来了一定的影响时, 他们便从“私 人领域” 转向 “公共领域”。因此粉丝作为公众, 在 通过粉丝群体的力量为社会献力的行为促使粉丝从 私人领域转向公共领域。

在此基础上, 粉丝与粉丝之间形成共同体, 产生 身份认同, 参与到公共事件当中。

就是我以前初中的时候看到了我同学，她拿了 一本TFBOYS 的杂志，我看了之后就是去萌生了去了 解他的想法，然后了解了解就喜欢上了。我跟这个 女的本来关系不好，是属于死对头的那种，因为她 
我喜欢上了易烊千胥，后来我们就成为了很好的朋 友，我们经常在一起讨论千胥。

( 访谈者：CC，访谈时间：2020 年 11 月 8 日)

通过原本不是朋友的同学喜欢上易烊千胥, 并且 两人成为好朋友, 还经常一起讨论关于千玺的事情。 她改变了原有的态度, 结交到了一个好朋友, 突破了 自我的社交边界, 在个体社交的私人领域转向相对的 公共领域, 与同为易烊千坌粉丝的人形成一个共同体。 这个共同体是对于粉丝个体而言的一种联系, 也是对 于粉丝群体的一种身份认同。

身份认同不仅体现在粉丝个体上, 还体现在粉丝 群体上。每个地区都有后援会, 一般在比较重要节日 或是偶像生日的时候都会举办线下活动, 例如包一家 餐厅, 或者是包一家影院, 把大家聚在一起为所喜欢 的偶像做应援。由此看来, 粉丝群体在一些重要节日、 偶像生日的时候参与线下的团建、应援活动, 促进了 粉丝之间的相互认识与联系, 同时加强了粉丝群体的 身份认同。这种身份认同归因于粉丝群体对同一个偶 像的喜欢, 这种喜欢促进了身份认同的产生, 通过线 下的应援活动得以加强和巩固。

除此之外, 微博用户@南羽千鹤在微博上公开发 布了一则微博, 上面提到:

从昨天凌晨开始的筹款预告，到昨天上午九点 开始的捐赠，为时四个小时，筹到二十五万多的捐 赠金额（鸟姐的公益心真的 [泪]搞哭我）。第一时间 报名了志愿者，有幸加入，真的真的特别开心檁县 能被注意到，能有幸以他的名义，帮助歌县灾区， 共渡难关。由筹集到的善款购置的第一批物资在今 天上午顺利抵达欱县深渡 ( 真的好快啊! 鸟姐速度 超快！）也超级幸运自己能作为粉丝志愿者到达灾 区帮忙，以他之名，做力所能及之事。

( 微博用户：南羽千鹤，发布时间：2020 年 7 月 11 日 15:53，来自 易烊千胥超话 )

从筹款、捐款再到线下志愿者, 这些行为行为是 她们作为粉丝, 将对易烊千胥的爱转化成一种对社会的 有益的行动, 她们通过易烊千胥粉丝的名义, 做自己力 所能及的事情, 帮助领县渡过难关, 她们认为这是幸运 的, 是开心的。这些都是身份认同形成的过程, 也是身 份认同的外在体现。身份认同的产生促使粉丝形成强相 互信任和强行动力, 在这次驰援疫区的行动中, 不只是 到达现场的粉丝志愿者, 还有许多易烊千胥粉丝通过互 联网平台捐款、收集物资, 甚至通过语言为需要帮助的
人打气。据不完全统计, 目前以“易烊千胥粉丝”名义开 展的疫区支援项目近 50 项, 总金额超过 136 万。社会 科学研究通常假设, 行动者行为的基础是理性认知, 是 对于利弊的权衡和效用的计算。但不强调情感也会有同 样的效果, 在这里我们将把捐款当成是一种情感的表演。 就捐款本身而言, 它就是一种实践, 但是这种实践是和 情感联系在一起的。我们可以通过语言来做事情, 通过 表达可以有一种实际的、社会的效果。粉丝群体的这样 一种情感, 实际上也是通过表达成为一种实践。情感表 达理论和情感实践理论强调情感一旦表达出来, 它就是 有社会结果的。

粉丝群体一起应援、做数据、参加公益等, 形成 一个 “共同体”, 为了一个共同的目标做努力。形成 共同体的过程就是粉丝之间相互认同的过程, 这种认 同促进了粉丝之间的信任, 形成了一种强相互作用力, 这种强相互作用力就是粉丝的身份认同, 粉丝个体将 这种身份认同外化至行动中, 从而又加强了粉丝间的 身份认同。情感可以被看作是一种策略, 当被定义为 一种行动方式时, 情感被呈现为社会结构的积极构成 者 [8], 这时粉丝群体便为共同体。

在网络民族志过程中, 我看到用户@易烊千胥 $\mathrm{V}$ 公益在微博上发布了这样一句话:

我很喜欢的一句话是“积少成多，聚爱成海"个 人的力量是有限的，但作为无数个粉丝组成的群体， 力量就会被放大无数倍。这一点也贯穿了本项目全 程：知道要支援疫区后，大家争先恐后伸出援手， 项目落地途中若遇到难题，粉丝们纷纷出谋划策， 身体力行地贡献点子。

( 微博用户：VogueMe，发布时间：2020 年 2 月 23 日 16:00)

他认为粉丝个体形成的共同体的行动力是很强的, 在每一次公益项目的过程中, 粉丝都会尽自己所能做力 所能及的事情, 这同样也是身份认同的外在体现。身份 认同不仅是共同体, 是群体之间的相互认同, 更是在这 种认同下的内部监督。每一次公益的进行, 物资的收集, 在粉丝群体身份认同下的内部监督, 保障了粉丝筹款的 透明、公正、公开。粉丝组织援助能够实行一场“内部 监督”督促公益项目落地, 实际帮助到了需要帮助的人 们, 是这次疫情捐款的成功原因、关键因素。

我之所以会相信他这个捐款，一个是因为平常 一直是这些人在做这些事，每一次捐款结束他们都 会出那种详细的明细，所以就是还是比较可信的。 
另外一个原因就是现在网络很发达，而且就是有很多 人都在盯着他们，这些都是不用担心的，因为大家其 实有参与到的人都会就是起到一个监督的作用。

( 访谈者：CC，访谈时间：2020 年 11 月 8 日 )

在公共领域下的身份认同的形成过程中, 易烊千 蛋发挥着极其重要的作用, 没有他成为“中介因素”, 粉丝的行为较难进入到公共领域的空间。由此可见， 在身份认同作为支撑的前提下, 粉丝在进行捐款时形 成了一种相互监督的共同体，可以让作为捐款者的粉 丝更能有效地监督募捐者是否适当且合法地将所募 集到的资金用到实处。养成类明星的粉丝群体为提高 明星的美誉度、知名度和社会认可度, 他们以偶像粉 丝甚至偶像的名义进行公益活动, 因此他们形成了一 个 “命运共同体”, 通过公益活动与社会大众的互动, 在这个过程中得到主流媒体的肯定性报道, 以及社会 大众对于这个粉丝及其偶像的认可, 从而使得粉丝群 体的身份认同从私人领域转向公共领域

\section{2. “共情” 机制的社会性表演}

粉丝在情感带动下所采取的的行动通常会考虑 到会给他们喜欢的偶像带来什么, 最后的结果由情感 产生的共情机制所作用, 这种情感反过来又制约了粉 丝的行为, 更好地推动了粉丝的行为从私人领域转向 公共领域。为了打破过去对于粉丝污名化的固有态度 和负面形象, 粉丝时常在语言中表达出他们所喜欢的 偶像为这个社会作出的有益贡献。

如果不是生日会上的一段 VCR，可能粉丝都不 会知道他从 14 岁起，就默默用零花钱资助偏远山区 的残疾小朋友。

( 微博用户：VogueMe，发布时间：2020 年 2 月 23

日 $16: 00)$

这种叙述表现了粉丝了对于易烊千胥做公益事 情的夸奖与肯定, 其情感的表达有利于粉丝将自己对 偶像的喜欢转化为对社会的有益的帮助, 进而将这种 情感体现为捐款这件事情。

用户@一易朵小 fafa 在微博上公开发布微博提 到:

自己一直都有在做公益比如老人小孩流浪猫狗 捐书捐衣服之类的活动，这也是当初喜欢上千千的 原因之一，但真的很幸运，天时地利人和给了我一 个可以代表自己喜欢的人（千千和鸟姐都是～）给
自己喜欢的城市一次帮助它的机会。

（微博用户：一易朵小 fafa，发布时间：2020 年 7 月 12 日 $12: 37$ 来自 易烊千胥超话)

由于易烊千笚和自己一样, 一直以来都参与公益, 所以喜欢上了易烊千疍, 她作为粉丝个体与自己所喜欢 的偶像产生身份、行动上的共鸣。又因为易烊千胥的原 因, 她能在这个社会需要帮助的时候, 以易烊千胥粉丝 的名义, 代表自己喜欢的偶像为这个社会贡献力量。这 就是粉丝的情感推动他们的行为从饭圈的私人领域转 向公共领域的一种表现。在与燕萍的访谈中她与我谈到:

他十分注重细节和仪式感，就在个人演唱会上 飘下的彩带都是千纸鹤的形状，演唱会外面的封闭 车厢，还有前两年扮演大白，就觉得这个人虽然话 不多但他都记在了心里。

(访谈者：燕萍，访谈时间：2020 年 11 月 19 日)

18 周岁成人礼演唱会上天空飘下的彩带是千纸 鹤的形状是他对粉丝爱的表现, 演唱会门口的封闭车 厢是他与粉丝之间的 “公开秘密”, 演唱会门口默默 扮演的大白是他关心粉丝的行动。这一些看似很普通 的事情, 却成为粉丝与易烊千胥之间最真切的情感交 流。从身份共鸣的产生, 到细节和仪式感的固粉, 粉 丝更加真实的 “参与” 到了偶像的生活, 相互陪伴, 共同成长。在这样一种双向输出的情感中, 粉丝个体 与养成系明星逐渐形成 “命运共同体”。情感是动机 结构的基础, 如需求、愿望和欲望, 将它们与行动和 符号系统联系起来 [8]。这种动机便促使粉丝积极主 动地捐款, 实际上背后是一种能动性 (agency) 的概 念。情感作为一个表述性、表演性的东西, 它是可以 造成一种实际的社会结果。这样的情感关系塑造了粉 丝的情感如何在身体和语言的维度表达, 并通过这些 表达进一步协商其社会互动 [9]。关于情感的语言被 用来在更广的社会情境中定位粉丝自己, 并在这些情 境中进行可能的行动 [10]。因此, 情感的表达有助于 粉丝群体生存的机会, 并且可以以适应环境需求的方 式粉丝群体的行为, 这种行为是粉丝的能动性及其情 感的表演性。在进行网络民族志的期间, 粉丝@南羽 千鹤在超话中发布了一则微博:

暴雨、涨水、高考延迟、无数的居民楼、车、 工业厂等等被泡，损失惨重。安徽一个小小的县城 被推上热搜，歌县。我很爱这个小县城，我应该做 点什么的吧，我又能做点什么呢，我好像做不了什 么。可是他却给了一个我机会，让我能为这个小县 城做点什么。 
( 微博用户：南羽千鹤，发布时间：2020 年 7 月 11

\section{日 15:53 来自 易烊千胥超话 )}

当 7 月洪灾袭击㪘县时, 粉丝因为易烊千先的原 因, 联手一起为当地做了一些能力范围内的事情, 将 对易烊千秃的喜欢转化成行动力一起帮助晾县渡过 难关。他们强调自己作为粉丝做公益的事情，因为他 们希望他们做公益都能做到实处，他们想通过自己做 公益让易烊千胥看到, 通过这样一种方式让千胥以粉 丝为骄傲。这种共情机制让粉丝作为公众出现，促使 了粉丝的行为从私人领域转向公共领域。如果 “情感” 本身作为一种理想类型, 无法被经验地观察, 我们仍 然能够观察到情感的社会性表达和展演 [11]。情感从 一种内在的感觉 (feeling) 转化成一种表达的行动, 这种表达是有事实的影响, 这种情感可以归纳为规范 性（normative）的情感理论。这种规范性路径在关 于文化的要素作为行为的指南发挥作用, 指导情感应 该如何在特定的情境中被表达, 以及一个人应该如何 感受, 感受什么以及何时感受 [9]。在此, 粉丝群体 创造了属于他们自己的一种 “情感领域”, 它是有着 自己的语言、规则、对象和边界的行动领域 [12]。

粉丝个体只是广大粉丝群体中的小小一员, 在这 次驰援疫区的行动中, 从散粉到粉丝站, 再从粉丝站 到粉丝站联合, 粉丝们希望他们将对偶像的喜欢转化 为为这个社会作贡献, 他们希望他们所做的事情不仅 可以为自己喜欢的明星树立公众形象, 为粉丝群体正 名, 也让喜欢的明星看到他的粉丝群体在追随他的路 上做出正能量, 积极向上的努力。因此, 粉丝个体与 养成系明星形成的 “命运共同体” 使得粉丝将这种 喜欢转化为以偶像的名义为这个社会做贡献, 进而促 使粉丝的行为从私人领域转向公共领域。这种共情机 制在由私到公的领域转向中扮演着重要的角色, 它可 以帮助粉丝在进入公共领域时保持情感与理性, 也可 以帮助社会大众对于粉丝公众产生情感上的共鸣, 更 有利于激发社会的关注。

\section{7. 结论}

本研究通过网络民族志和深度访谈的方式, 对养 成系明星的粉丝群体的捐款公益行为进行了探索性 的研究。研究发现由于养成系明星的“桥梁”的作用, 粉丝围绕在养成系明星周围形成了 “共同体” 网络, 因为养成系明星这个核心的存在, 粉丝得以把个人诉 求或行为以 “共同体” 的方式表达出来而变成了集体 行动。并且, 在 “共同体” 网络的支持下, 他们将粉 丝对于明星之间的私人情感性 “共情” 转变成集体性 的对于社会公益的“共情”。“共情”存在于 “共同体” 之中, “共情” 使得 “共同体” 变得更团结、更有力 量, 两者相互依赖、相互构成, 彼此互动, 最终促成 了捐款行为发生。综上所述，养成系粉丝在身份认同 的机制下由私人领域到公共领域的结构转型机制就 是粉丝 “共同体” 的形成和社会 “共情” 的产生。
研究发现粉丝形成的“共同体”对于产生依托于 共同体产生的 “共情” 至关重要，这种情感可以让粉 丝团结起来去做一些有益于公共社会的事情。那么, 为了他们可以更多的去做这样的事情, 我们需要采取 的措施是要去培育、完善、监管这样一个“共同体”。 要让粉丝自发的、自愿的去为一个公序良俗的事情去 奋斗, 我们需要让这个 “共同体” 更加团结, 更加富 有情感和社会 “共情” 能力。

\section{REFERENCES}

[1] Habermas, J. (1992). Public Sphere and Private Life: Toward a Synthesis of Current Historiographical Approaches to the Old Regime.

[2] Jiaying, Y. The reinterpretation of Habermas's "public sphere" theory in the contemporary Chinese context[J]. Journalism and Communication, 2019(14):4-6+9.

[3] Guanglei, S. An analysis of the superposition of public and private spheres in social media from acceptance theory[J]. Journal of Journalism Research, 2020, 11(13):24-25.

[4] Zhang W. The Internet and new social formation in China: Fandom publics in the making[M] Routledge, 2016.

[5] Yutian, S. Research on the Fan Culture of "Foster" Idols [D]. Jinan University, 2016.

[6] Yefeng, C. Research on the Fan Culture of "Foster" Idols in the New Media Era [D]. Huazhong University of Science and Technology, 2018.

[7] Huiying S, Chaoqiong, M. Analysis of social emotional value of hot events in the public sphere[J]. Modern Communication (Journal of Communication University of China), 2020, 42(07):147-152.

[8] Turner, J. et al. The Sociology of Emotion [M]. Shanghai: Shanghai People's Publishing House, 2007.10

[9] Stets, J.E. and Turner, J.H. eds. Handbook of the sociology of emotions: Volume II [M]. Springer, 2014. 225

[10] Stets, J.E. and Turner, J.H. eds. Handbook of the sociology of emotions: Volume II [M]. Springer, 2014. 230

[11] Stets, J.E. and Turner, J.H. eds. Handbook of the sociology of emotions: Volume II [M]. Springer, 2014. 221.

[12] Stets, J.E. and Turner, J.H. eds. Handbook of the sociology of emotions: Volume II [M]. Springer, 2014. 226. 